\title{
The Influence of Host Genotypes on the Endophytes in the Leaf Scar Tissues of Apple Trees and Correlation of the Endophytes with Apple Canker (Neonectria ditissima) Development
}

\author{
Matevz Papp-Rupar, Amanda Karlstrom, Thomas Passey, Greg Deakin, and Xiangming $\mathrm{Xu}^{\dagger}$ \\ NIAB EMR, New Road, East Malling, West Malling, Kent, ME19 6BJ, U.K. \\ Accepted for publication 7 February 2022.
}

\section{ABSTRACT}

Bacterial and fungal endophytes may help their host in terms of improved tolerance to abiotic and biotic stresses and enhanced growth. European apple canker, caused by Neonectria ditissima, is widespread in apple-growing regions. Infection by $N$. ditissima occurs through artificial or natural wounds, including leaf scars, picking wounds, and pruning cuts. Using $F_{1}$ progeny trees in an experimental orchard derived from a cross between a canker-susceptible genotype and a canker-tolerant or -resistant genotype, we assessed both bacterial and fungal endophyte communities in apple leaf scars, and determined correlations of endophytes with canker development. All trees were artificially inoculated with an $N$. ditissima isolate postplanting. Specific components of apple endophytes as well as a number of individual fungal or bacterial groups in leaf scars were partially genetically controlled by host genotypes. Several bacterial groups were significantly correlated with canker-related traits, mostly positively associated with canker tolerance. A few fungal groups may facilitate canker development whereas others may compete with canker. However, most of these microbial groups could not be identified to the species level with confidence; even for those groups which could be assigned to the species level, there is insufficient knowledge about their ecological characteristics in relation to plants. The present results may be used to inform further research using biocontrol to manage N. ditissima and breeding for resistance.

Keywords: apple endophytes, crop, European apple canker, heritability, leaf scars, Neonectria ditissima, plant pathology
Many bacterial and fungal endophytes inhabit plant tissues without causing any adverse effect and may benefit their host in terms of improved tolerance to abiotic and biotic stresses (e.g., pests and pathogens) and enhanced growth (Dini-Andreote 2020; Khare et al. 2018; Omomowo and Babalola 2019). Due to increasing public concern about using pesticides, increased incidence of pesticide resistance, and decreasing numbers of commercial pesticides available (primarily due to regulation), there is an urgent need to exploit

'Corresponding author: X. Xu; xiangming.xu@niab.com

Funding: This work was supported by the U.K. Biotechnology and Biological Science Research Council (grant number BB/P007899/1 and BB/P000851/1) and several industry organizations: the Agriculture and Horticulture Development Board, Adrian Scripps Limited, Avalon Produce Limited, ENZA (T\&G global subsidiary), Frank P Matthews Limited, and Worldwide Fruit Limited.

*The $e$-Xtra logo stands for "electronic extra" and indicates that supplementary figures are published online.

The author(s) declare no conflict of interest.

This article is in the public domain and not copyrightable. It may be freely reprinted with customary crediting of the source. The American Phytopathological Society, 2022 beneficial microorganisms, including endophytes, and microbialderived compounds for disease management.

Neonectria ditissima is a fungal pathogen that causes wood cankers in apple and other broad-leaved trees (Saville and Olivieri 2019; Weber 2014). Canker is widespread in apple-growing regions in most of the temperate areas of the world. $N$. ditissima infects trees through artificial or natural wounds; most frequently, through leaf scars, wounds caused by picking fruit, and pruning cuts. A most damaging phase of this disease is that latent infection established in nurseries can develop rapidly on the main stem postplanting, killing young trees. Due to withdrawal of several effective fungicides (e.g., carbendazim and copper-based products), current chemical control strategies only have a limited effect on the establishment and spread of $N$. ditissima. Thus, host resistance is an important component in managing canker disease. Unfortunately, many modern apple varieties are derived from a narrow genetic background with a high susceptibility to apple canker (GómezCortecero et al. 2016). High-density orchards further exacerbate this problem because canker lesions on the main stem can easily girdle and subsequently kill small trees postplanting in such an orchard. Host resistance against $N$. ditissima is of a quantitative nature (Bus et al. 2019; Gómez-Cortecero et al. 2016). Therefore, it may take a long time to breed apple cultivars with effective 
resistance against the pathogen given the nature of perennial trees and quantitative resistance.

There is evidence of host-genotypic effects on endophyte communities in apple (Hirakue and Sugiyama 2018; Liu et al. 2018, 2020), influencing both species richness and composition. Although the endophytic microbe composition was only studied in a limited number of apple cultivars, the results indicate that cultivars with a higher degree of relatedness also share similarities in endophyte community (Liu et al. 2018). Apple resistance to leaf pathogens appears to correlate more with fungal endophytes than bacterial endophytes (Hirakue and Sugiyama 2018). In addition to cultivar, specific location and apple tissue type can also considerably influence endophyte composition (Liu et al. 2020; Olivieri et al. 2021a).

It has been demonstrated recently that a number of apple endophytes at leaf scars were associated with the host susceptibility to $N$. ditissima (Olivieri et al. 2021a). Specific endophytes from apple showed in vitro antagonistic effects against $N$. ditissima (Liu et al. 2020). Thus, manipulating apple endophyte communities may be a viable approach to manage canker disease. Apple endophyte composition could be altered through specific agronomic practices or augmented application of specific endophyte strains with biocontrol ability to improve host resistance or tolerance against $N$. ditissima. Another approach would be to include specific (desirable) endophytes as a selection criterion in breeding programs to breed cultivars with desirable endophyte composition or the ability to recruit these specific microbes for disease suppression or tolerance. To adopt this second approach, we need to assess the magnitude of the heritability of endophyte communities with respect to overall communities as well as specific endophytes. Such knowledge of the host genetic component of endophytes is lacking because the current research has been focusing on the determination of differences in endophytes between genotypes.

In this study, we estimated the broad-sense heritability of endophyte communities as well as individual endophytes in apple leaf scars, an important entry site for $N$. ditissima. Progeny derived from a cross between two genotypes (one canker-susceptible cultivar and the other a canker-tolerant cultivar) were evaluated under field conditions. Bacterial and fungal endophytes at the leaf scar tissues were profiled with amplicon sequencing and correlated with canker development. All sequences have been deposited in the European Nucleotide Archive under project reference PRJEB49633.

\section{MATERIALS AND METHODS}

Field design and layout. A cross between cultivars 'Aroma' and 'Golden Delicious' was made in 2015. Canker resistance is not well understood yet but it is quantitative in nature. The two parents were selected based on empirical evidence as well as limited lab artificial-inoculation data (Gómez-Cortecero et al. 2016), which classified the two parents as moderately susceptible and highly resistant or tolerant, respectively, to $N$. ditissima. All $70 \mathrm{~F}_{1}$ genotypes in the family, including the parents, were grafted onto M9 EMLA rootstocks in January 2017 at East Malling, United Kingdom. The plants were maintained in pots in polytunnels until October 2017, when they were planted in a randomized block design in an orchard. There were four blocks, with one tree per genotype in each block.

Inoculation with $N$. ditissima. The trees were artificially inoculated with a single isolate of $N$. ditissima $(\mathrm{Hg} 199)$ at leaf scars in November 2018 in order to eliminate the issue of inoculum heterogeneity in the orchard. Because the research objective was to compare resistance and susceptibility of genotypes to $N$. ditissima, we decided to eliminate the issue of field inoculum heterogeneity via artificial inoculation from complicating data interpretation.
The inoculum was prepared according to a published protocol (Gómez-Cortecero et al. 2016). Five artificial leaf scars were inoculated per tree, and each leaf scar (pseudoreplicate) was positioned on a separate branch. A droplet of $6 \mu \mathrm{l}$ of spore suspension $\left(10^{5}\right.$ macroconidia/ml) was pipetted onto each artificial leaf scar wound, then covered with petroleum jelly (Vaseline) immediately after absorption. The petroleum jelly was removed 2 weeks after inoculation.

Each inoculated leaf scar was marked to allow repeated measurements over time. Canker lesion development was measured with a digital caliper at three time-points: 5, 8, and 11 months postinoculation (mpi). A final assessment was conducted at 20 mpi on the percent branch area with foliage, percent branches with cankers, and number of cankers. Average number of cankers per branch was then calculated for each tree.

Sampling leaf scars, DNA extraction, and sequencing. Many trees died of canker before leaf scars were sampled in autumn 2019 before leaf fall. Only those genotypes with a minimum of three surviving trees were sampled. In total, we sampled 216 trees, including the two parents and $54 \mathrm{~F}_{1}$ genotypes. Leaf scars were sampled for characterization of endophytes in leaf scar tissues, following a published protocol (Olivieri et al. 2021a). Briefly, sampling and subsequent sample processing consisted of the following steps. Four 20-cm-long, healthy (i.e., free of any disease symptoms on all leaf scars), 1-year-old extension shoots were sampled randomly from each tree, not including those inoculated shoots (because those already became 2-year-old shoots). Our previous study showed that $N$. ditissima is unlikely to be systemic but usually is confined within the vicinity of canker lesions (Olivieri et al. 2021b). Thus, N. ditissima is not expected to be present in these healthy leaf scars because new infections of leaf scars usually take place after leaf fall in autumn. Three to four freshly exposed leaf scars were sampled from each of the four shoots and pooled into one sample for a single tree. Samples were freeze dried and dry weight was recorded before homogenization with a Geno/ Grinder 2010 (SPEX CertiPrep) for $2 \mathrm{~min}$ at 1,500 rpm using 2-ml tubes and two 5-mm steel ball bearings. Sterile $0.1 \mathrm{M}$ phosphatebuffered saline (PBS) buffer was added to the homogenized samples at a 1:5 ratio (dry weight [milligrams]/volume [microliters]). DNA was extracted from $120 \mu$ of PBS resuspended homogenate with the DNeasy Plant mini kit (Qiagen) according to the manufacturer's protocol. Pure DNA was eluted from the spin columns in two steps, each using $50 \mu \mathrm{l}$ of molecular-grade water and $1 \mathrm{~min}$ of incubation before spinning at $14,000 \times g$. The purity and quantity of extracted DNA was assessed with NanoDrop1000 (Thermo Fisher Scientific). DNA extraction of samples was repeated if their concentration did not exceed $10 \mathrm{ng} / \mu \mathrm{l}$. The samples were shipped on ice to Novogene UK (Cambridge, U.K.) where PCR amplification, library prep, and metabarcoding sequencing was done. The target regions were internal transcribed spacer (ITS)1-1F (ITS1-1F-F: 5'-CTTGGTCATTTAGAGGAAGTAA-3', ITS1-1F-R: 5'-GCTG CGTTCTTCATCGATGC-3') and 16S V5-V7 (799F: 5'-AACM GGATTAGATACCCKG-3', 1193R: 5'-ACGTCATCCCCACCTT $\left.\mathrm{CC}-3^{\prime}\right)$. To reduce untargeted amplification of plant mitochondrial and plastid DNA with the $16 \mathrm{~S}$ amplicon, we used mPNA (5'-ggc aagtgttcttcgga- $\left.3^{\prime}\right)$ and pPNA (5'-ggctcaacctggacag- $\left.3^{\prime}\right)$ oligo bloc kers (PNA Bio) at $200 \mathrm{nM}$ final concentration. Samples were sequenced on Illumina NovaSeq platform in the 250-nucleotide paired-end mode.

We estimated fungal and bacterial community sizes with quantitative PCR (qPCR). Briefly, a pooled sample was prepared combining $2 \mu \mathrm{l}$ of extracted DNA from each sample. Calibration curve (six steps, 10-fold serial dilutions) was prepared using the pooled sample, aliquoted, and stored at $-20^{\circ} \mathrm{C}$. Each block of samples was 
run on a separate 384-well plate in at least two dilutions (5x and $20 \times$ ), each dilution in duplicate. The standard curve was run in triplicate alongside samples and a nontemplate control on each plate. Reactions (10 $\mu \mathrm{l}$ each) were performed with SsoAdvanced Universal SYBR Green Supermix (Bio-Rad) and $2 \mu \mathrm{l}$ of template DNA. The same primers as in the metabarcoding sequencing were used (ITS1-1f and 16S V5-V7) at a final concentration of $100 \mathrm{nM}$. PNA primers (mPNA and pPNA) at $200 \mathrm{nM}$ final concentration were used in combination with $16 \mathrm{~S}$ V5-V7 to prevent amplification of mitochondrial and chloroplast contaminants. The cycling protocol was denaturation for $5 \mathrm{~min}$ at $94^{\circ} \mathrm{C}$ and 40 cycles of $60 \mathrm{~s}$ at $94^{\circ} \mathrm{C}$, $10 \mathrm{~s}$ at $75^{\circ} \mathrm{C}, 30 \mathrm{~s}$ at $55^{\circ} \mathrm{C}$, and $60 \mathrm{~s}$ at $72^{\circ} \mathrm{C}$, followed by melt curve analysis.

Community size in terms of ITS or 16S copy number was estimated using a calibration curve for each plate separately. First, we calculated the efficiency of amplification for each sample and standard curve on each plate separately $\left[\mathrm{E}=10^{(-1 / \text { slope })}\right]$. Efficiency of calibration curves and samples were set to $100 \%$ by correcting their raw quantification cycle $(\mathrm{Cq})$ values with their respective efficiency using the following formula: $\mathrm{Cq}_{\mathrm{c}}=\log _{2}\left(\mathrm{E}^{\mathrm{Cq}}\right)$. The corrected $\mathrm{Cq}$ $\left(\mathrm{Cq}_{\mathrm{c}}\right)$ value of a sample was used to calculate its theoretical copy number present in each sample using the efficiency corrected calibration curve equation: $\mathrm{C}=\left(\mathrm{Cq}_{\mathrm{c}}-\mathrm{I}_{\mathrm{c}}\right) / \mathrm{S}_{\mathrm{c}}$, where $\mathrm{C}$ represents estimated $\log _{10}$ copy number of ITS or $16 \mathrm{~S}$ in the sample, $\mathrm{Cq}_{\mathrm{c}}$ represents efficiency corrected $\mathrm{Cq}$ value of the sample, and $\mathrm{I}_{\mathrm{c}}$ and $\mathrm{S}_{\mathrm{c}}$ represent intercept and slope, respectively, of the efficiency corrected calibration curve.

The samples with efficiency below 0.8 and above 1.2 were repeated at lower DNA dilution $(10 \times$ and $40 \times$ or $20 \times$ and $100 \times)$ to minimize the effect of PCR inhibitors. Samples that failed the efficacy threshold were removed from further analysis. The mean of four technical repeats on each sample was used.

Sequencing processing and taxonomy assignment. Pairedend amplicon sequence data were processed to produce operational taxonomic unit (OTU) representative sequences and frequency tables. Read pairs containing incorrect bases in primer regions or less than 250 bases in both pairs were discarded. Remaining read pairs were merged with a maximum difference in overlap of $5 \%$ with the UPARSE pipeline (version 10.0) (Edgar 2013). Merged reads of $<250$ and $400 \mathrm{bp}$ for ITS and $16 \mathrm{~S}$, respectively, were discarded. All remaining (unfiltered) reads were retained. Unfiltered reads were then filtered for quality, with a maximum expected error threshold of 0.5 per sequence (Edgar and Flyvbjerg 2015). Forward and reverse primers were removed from both filtered and unfiltered datasets. Filtered reads were dereplicated and singletons were discarded; then, representative OTUs at $97 \%$ similarity were constructed with the UPARSE pipeline. UPARSE also removes suspected chimeral sequences. Then, the unfiltered reads were aligned the OTU representative sequences at the level of $97 \%$ similarity to produce OTU frequency tables. Finally, the SINTAX algorithm (https://www. drive5.com/usearch/manual/sintax_algo.html) was used to assign taxonomic ranks to the OTU representative sequences with the Unite V8.3 (2021-05-10) fungal database (Kõljalg et al. 2013) and the RDP training set 18 bacterial database (Cole et al. 2014). OTUs which were identified as chloroplast and mitochondria were then removed before statistical analysis.

Statistical data analysis. Only the most abundant OTUs that accounted for $99.0 \%$ of the total counts were retained for statistical data analysis. Rare taxa were excluded from statistical analysis for several reasons. First, the present objective was to assess the extent of differences between genotypes. However, genotypes would unlikely differ in the relative abundance of rare taxa (given their low counts). For the same reason, correlation of these rare taxa with canker variables also would not be expected to be significant.
Second, these rare taxa had very little effect on the main characteristics of microbiome composition, represented by principal components (PCs). Finally, the abundance of these rare taxa is more prone to sequencing errors than for the other taxa.

Before statistical analysis, the fungal and bacterial OTU tables were normalized by two methods: by the qPCR values of the generic ITS or $16 \mathrm{~S}$ primers, or by the median-of-ratios (MR) method implemented in DESeq2 (Love et al. 2014). The former normalization incorporates the amount of microbial biomass between samples, estimated as the total number of DNA copies per sample, as well as relative proportion of individual OTUs within a sample. The latter only considers the relative proportions of individual OTUs within a sample. The MR method was used because of (i) nonsignificant differences between genotypes in qPCR values of $16 \mathrm{~S}$ and ITS and (ii) large variability in qPCR values between replicates within each genotype.

Both $\alpha$ - and $\beta$-diversity indices were calculated. The former measures within-sample diversity in term of the number of species present and their corresponding abundance, whereas the latter measures between-sample diversity. Two $\alpha$-diversity indices (Shannon and Simpson) were calculated with the $\mathrm{R}$ vegan 2.3-1 package (Dixon 2003). The ranks of $\alpha$-diversity indices were subjected to analysis of variance to assess the differences between the two parents and between progeny genotypes via a permutation test for statistical significance. The $\beta$-diversity indices were calculated as Bray-Curtis indices, then subjected to permutational multivariate analysis of variance between parents and between progeny genotypes with a permutation test based on pseudo- $F$ ratios (implemented as the Adonis function in the vegan package).

The main objective of the present study was to determine whether there is significant genetic variability among $F_{1}$ progeny genotypes. This was achieved by a random effect model in which the total variability among $\mathrm{F}_{1}$ genotypes was partitioned into environmental $\left(V_{E}=\right.$ between blocks + residual $)$ and genetic $\left(V_{G}=\right.$ between $\mathrm{F}_{1}$ genotypes) variability. The significance of genetic variability (component) was statistically tested by comparisons of two nest models with a $\chi^{2}$ test with one degree of freedom: one with the genotypic component included and the other without. The broad-sense heritability was then estimated as $V_{G} /\left(V_{E}+V_{G}\right)$.

These variance components were estimated with the lmer function in the lme4 package (Bates et al. 2015). For each normalized set of counts data, two types of data were used for estimation of the genetic components: PC scores, representing the overall microbial composition, and the normalized counts data of those OTUs with highest count values (Table 1). Before PC analysis, the normalized counts data were logarithm transformed on the natural base, then standardized. Similarly, normalized OTU count values were logarithm transformed on the natural base before analysis. The random effect model was fitted to the data with the $\mathrm{R}$ lme4

TABLE 1

Number of the principal components (PCs) and operational taxonomic units (OTUs) with the highest counts for inclusion in estimation of differences between $F_{1}$ genotypes and correlation with canker variables

\begin{tabular}{lcccccc}
\hline & \multicolumn{2}{c}{$\begin{array}{c}\text { Quantitative } \\
\text { PCR normalized }\end{array}$} & & \multicolumn{2}{c}{$\begin{array}{c}\text { Median of ratio } \\
\text { normalized }\end{array}$} \\
\cline { 2 - 3 } Group & PCs & OTUs & & PCs & OTUs \\
\hline $\begin{array}{l}\text { Fungi (internal transcribed } \\
\text { spacer) }\end{array}$ & 30 & 100 & & 80 & 100 \\
\hline Bacteria (16S) & 30 & 200 & & 50 & 200 \\
\hline
\end{tabular}


package (Bates et al. 2015). Within the analysis of each data type (PC or OTU), the Benjamini-Hochberg (BH) adjustment (Benjamini and Hochberg 1995) was applied to correct for the false discovery rate associated with multiple testing. Statistical significance was determined at the 5\% level (BH adjusted). In addition to the estimation of broad-sense heritability, correlation (both Pearson and Spearman) of canker-related variables with PC scores and normalized OTU counts were calculated.

All statistical analyses were carried out in $\mathrm{R}$ 4.0.3 ( $\mathrm{R}$ Core Development Team 2019).

\section{RESULTS}

Overall sequencing, OTU generating, and qPCR results. Fungi. In total, there were 4,268 fungal OTUs, most of which had few reads. The top 6 and 34 most abundant OTUs accounted for over 50 and $90 \%$ of the total reads, respectively (Fig. 1A). The top 206 OTUs accounted for $99.0 \%$ of total reads and were included in subsequent analysis.

The number of reads assigned to OTUs in each sample ranged from 51,780 to 134,300 , with a median of 117,676 ; the number of reads for each OTU ranged from 1,706 to 4,289,841, with a median of 7,532. The top two were both identified as Filobasidium spp.: $F$. wieringae and $F$. chernovii, accounting for 18.0 and $12.0 \%$ of the total reads, respectively. The OTUs with the third and fourth most reads were both identified as Vishniacozyma spp., jointly accounting for $13.0 \%$ of the total reads. Of the 206 fungal OTUs, only $137,118,104,79,60$, and 34 could be assigned to the taxonomic rank of phylum, class, order, family, genus, and species, respectively, with $>80 \%$ confidence. Basidiomycota and Ascomycota accounted for 59.3 and $15.8 \%$, respectively, of the total reads, whereas $24.9 \%$ of the reads could not be assigned to a phylum with $>80 \%$ confidence and, hence, were designated as unknown (Fig. 2A).

For 13 of the 216 samples ( $54 \mathrm{~F}_{1}$ genotypes and the two parents), we failed to obtain reliable qPCR ITS values. Of the remaining samples, there were large variabilities in the ITS qPCR values among replicates within a given genotype (Fig. 3A); genotypes did not differ significantly in the ITS qPCR values.
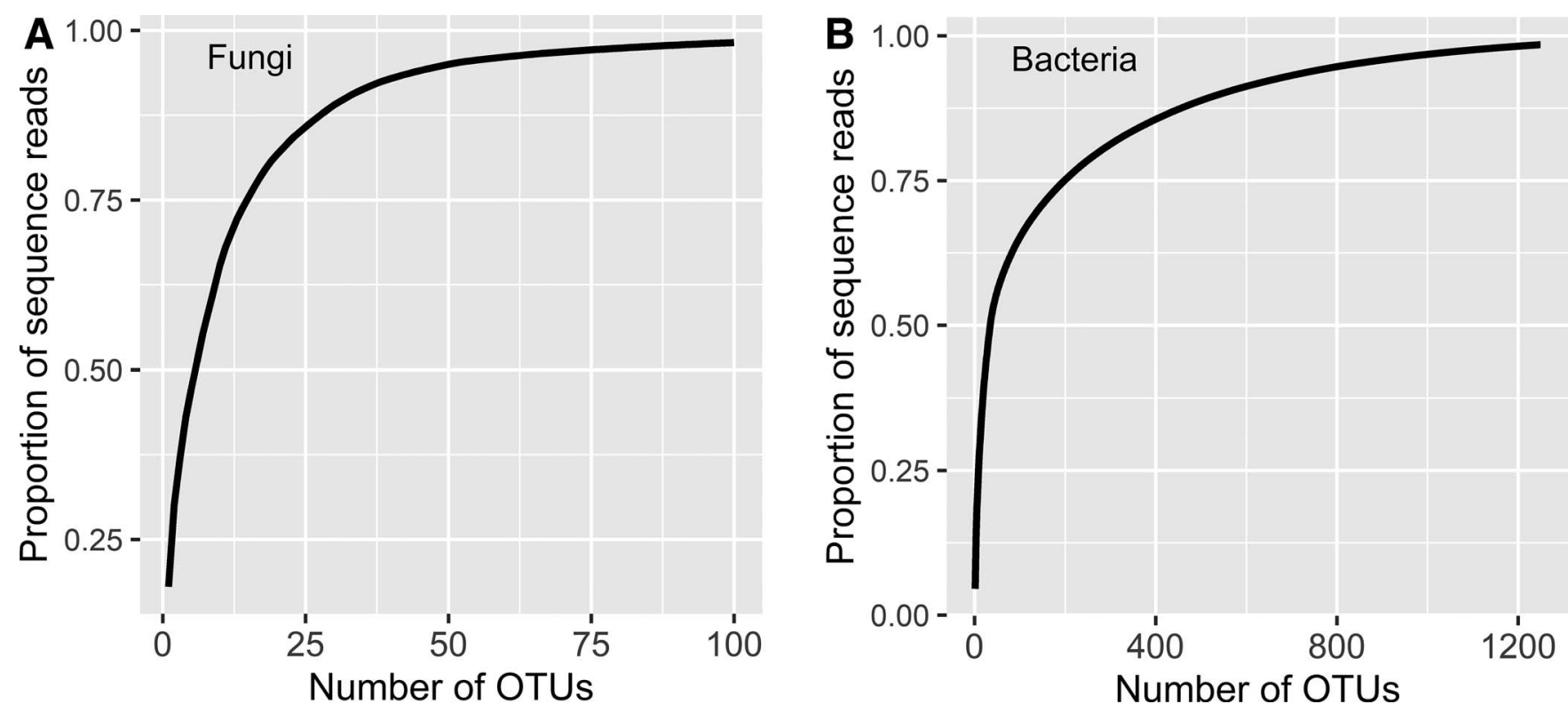

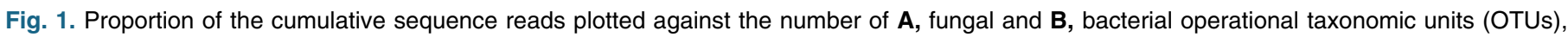
where the OTUs were sorted in the descending order with respect to the number of their sequence reads. 
Genetic components of PC scores. The summary of results for PC scores is shown in Table 2.

Fungi. For the qPCR-normalized data, the first two PCs explained approximately 50.9 and $4.0 \%$ of the total variability (Supplementary Fig. S1A). $\mathrm{F}_{1}$ genotypes differed $(P<0.001)$ for PC5, with the corresponding estimated heritability value of $27.5 \%$. For the MR-normalized data, the first two PCs explained only approximately
9.3 and $7.4 \%$ of the total variability (Supplementary Fig. S1B). $F_{1}$ genotypes differed for PC1, PC4, P9, PC12, P17, and P44, with corresponding heritability estimates of $5.5,33.7,20.8,23.4,19.4$, and $21.9 \%$.

Bacteria. For the qPCR-normalized data, the first two PCs explained approximately 45.3 and $3.8 \%$ of the total variability (Supplementary Fig. S2A). Only for 2 (PC3 and PC8) of the top 30 PCs
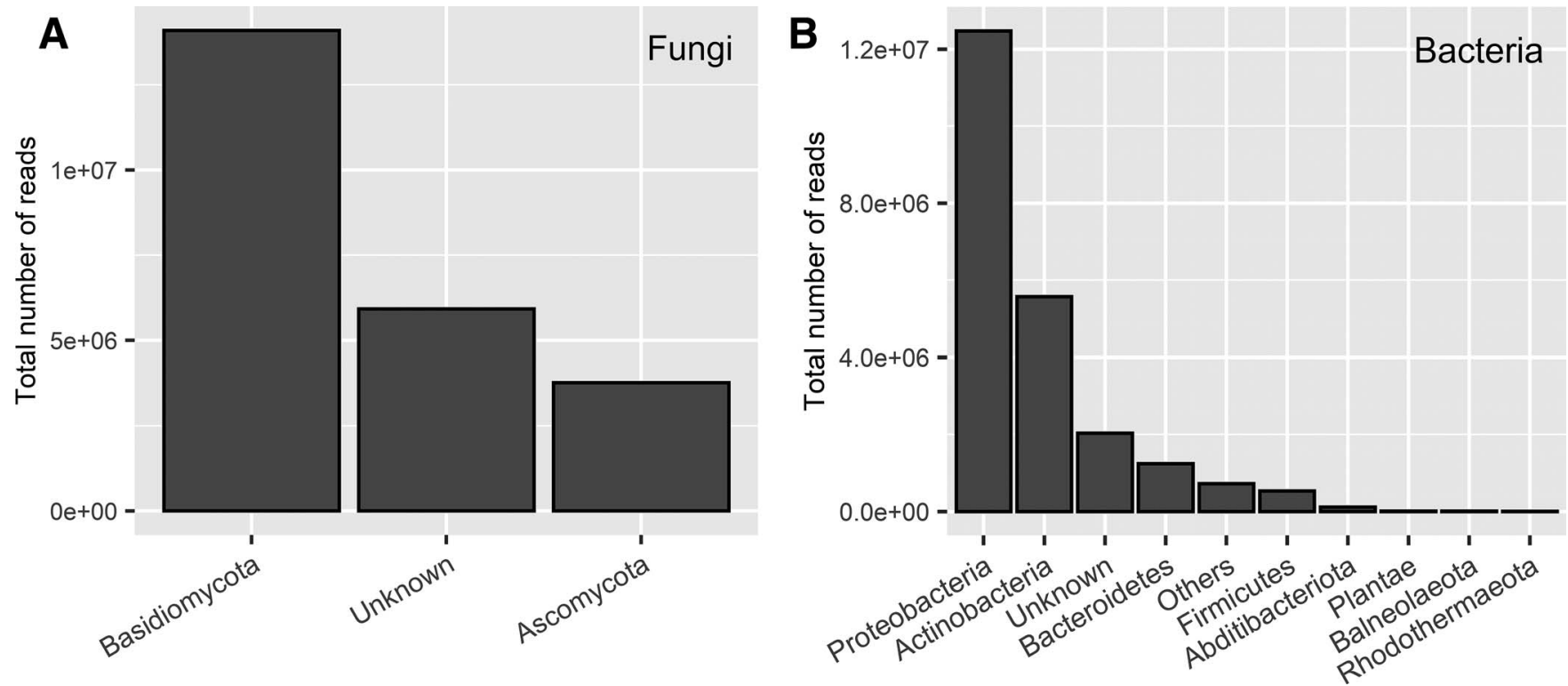

Fig. 2. Histogram of the number of the A, internal transcribed spacer and $\mathbf{B}, 16 \mathrm{~S}$ sequences that were assigned to the phylum at the $80 \%$ confidence level; the "Unknown" group consists of those operational taxonomic units that cannot be assigned to a unique phylum.
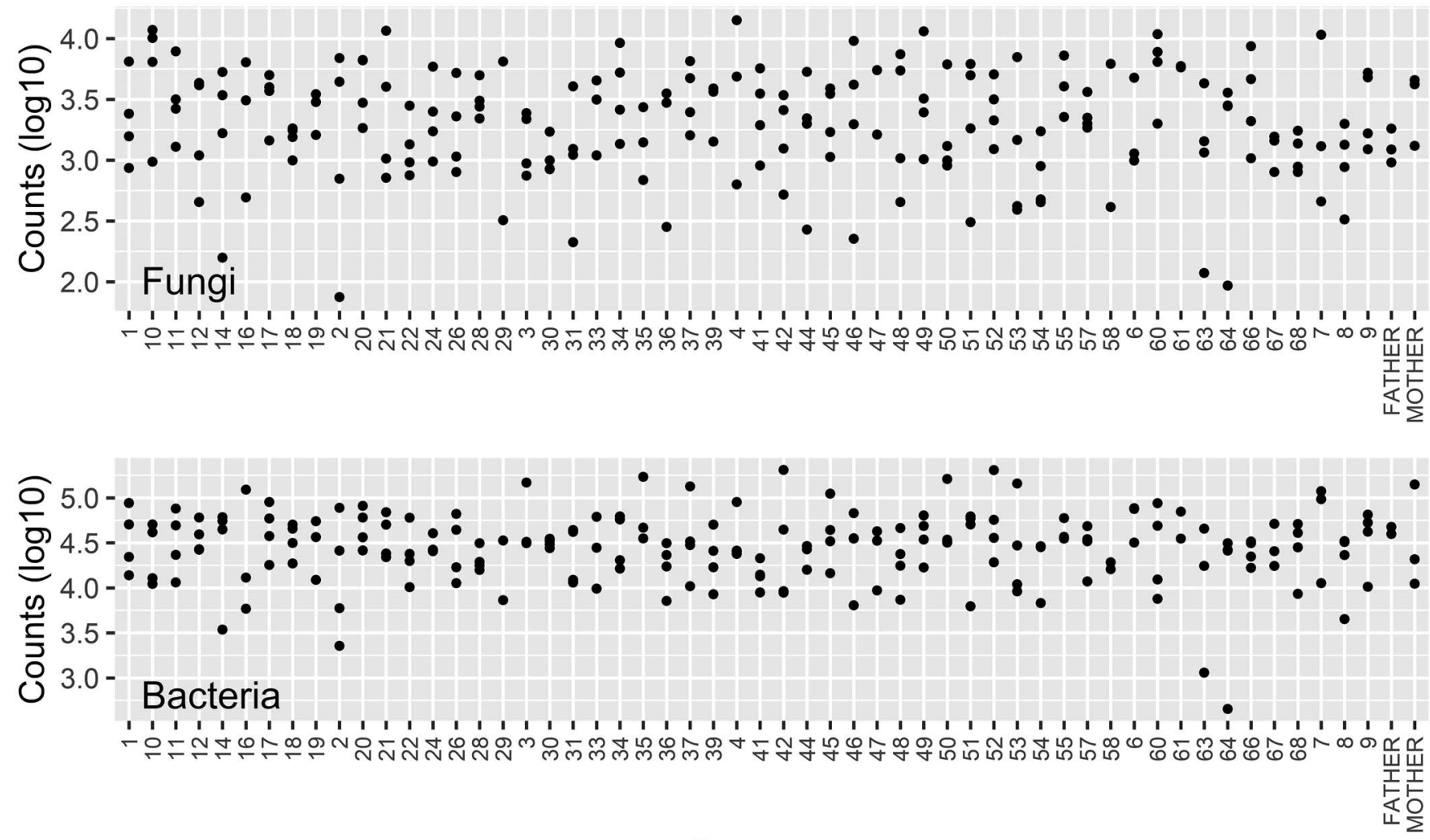

\section{Genotypes}

Fig. 3. Quantitative PCR results of both the internal transcribed spacer and $16 S$ of endophytes in apple leaf scar tissues of individual trees of $F_{1}$ and parental genotypes. The cross was between cultivars Aroma and Golden Delicious. 
did the $54 \mathrm{~F}_{1}$ genotypes differ significantly $(P<0.05)$, with estimated heritability of 29.5 and $30.0 \%$, respectively. For the MR-normalized data, the first two PCs explained only approximately 9.2 and $8.0 \%$ of the total variability (Supplementary Fig. S2B). For 7 (PC2, PC7, PC8, PC16, PC17, and PC26) of the top $50 \mathrm{PCs}, \mathrm{F}_{1}$ genotypes differed significantly $(P<0.05)$, with heritability estimates ranging from $14.5 \%$ (PC13) to $33.2 \%$ (PC7).

Genetic components of individual OTUs. The summary of results for individual OTUs is shown in Table 2.

Fungi. For the qPCR-normalized data, $\mathrm{F}_{1}$ genotypes did not differ significantly in any of the top 100 OTUs. For the MR-normalized data, $\mathrm{F}_{1}$ genotypes differed $(P<0.05)$ for 24 of the top 100 OTUs (Fig. 4A; Table 3). Only 7 of the 24 OTUs could be assigned to the species level; 2 of these OTUs were both identified as Tilletiopsis washingtonensis. The estimate of broad-sense heritability ranged from 10.2 to $34.7 \%$, with an average of $18.5 \%$.

Bacteria. For the qPCR-normalized data, $\mathrm{F}_{1}$ genotypes differed significantly in only 1 of the top 200 OTUs (OTU89, Roseomonas sp.), with an estimated broad-sense heritability of $27.9 \%$. For the MR-normalized data, $F_{1}$ genotypes differed significantly for 47 of the top 200 OTUs (Fig. 4B; Table 4). The estimate of broad-sense heritability ranged from 8.1 to $37.3 \%$, with an average of $18.5 \%$. These 47 OTUs also included OTU89; indeed, there were other OTUs from Roseomonas spp.

Canker development. Average canker size over the first three measurements (month 5, 8, and 11) ranged from 4.6 to $23.5 \mathrm{~cm}$,

TABLE 2

Number of cases where there was significant genetic variability in the principal components (PCs) and top fungal or bacterial operational taxonomic units (OTUs) or correlation of endophytes with canker variables ${ }^{\mathrm{a}}$

\begin{tabular}{|c|c|c|c|c|c|c|c|c|}
\hline \multirow[b]{2}{*}{ Variables } & \multicolumn{4}{|c|}{ Quantitative PCR normalized } & \multicolumn{4}{|c|}{ Median of ratio normalized } \\
\hline & PCs & OTUs & PCs & OTUs & PCs & OTUs & PCs & OTUs \\
\hline Genetic variance & 1 & 0 & 2 & 1 & 6 & 24 & 7 & 47 \\
\hline \multicolumn{9}{|l|}{ Correlation } \\
\hline \multicolumn{9}{|l|}{ Individual based } \\
\hline Canopy & 1 (1) & $2(0)$ & $1(1)$ & $0(0)$ & $1(1)$ & $7(7)$ & $1(1)$ & $6(0)$ \\
\hline Shoots cankered (\%) & $0(0)$ & $0(0)$ & $0(0)$ & $0(0)$ & $0(0)$ & $0(0)$ & $0(0)$ & $0(0)$ \\
\hline \multicolumn{9}{|l|}{ Family based } \\
\hline Canker size & $1(1)$ & $0(2)$ & $0(0)$ & $2(2)$ & $1(1)$ & $2(2)$ & $2(2)$ & $0(0)$ \\
\hline Canopy & $1(0)$ & $5(0)$ & $0(0)$ & $0(0)$ & $1(0)$ & $8(6)$ & $1(0)$ & $4(10)$ \\
\hline
\end{tabular}

${ }^{a}$ Values, shown as $x(y)$, indicate the $x$ and $y$ number of significant Pearson and Spearman correlations, respectively.
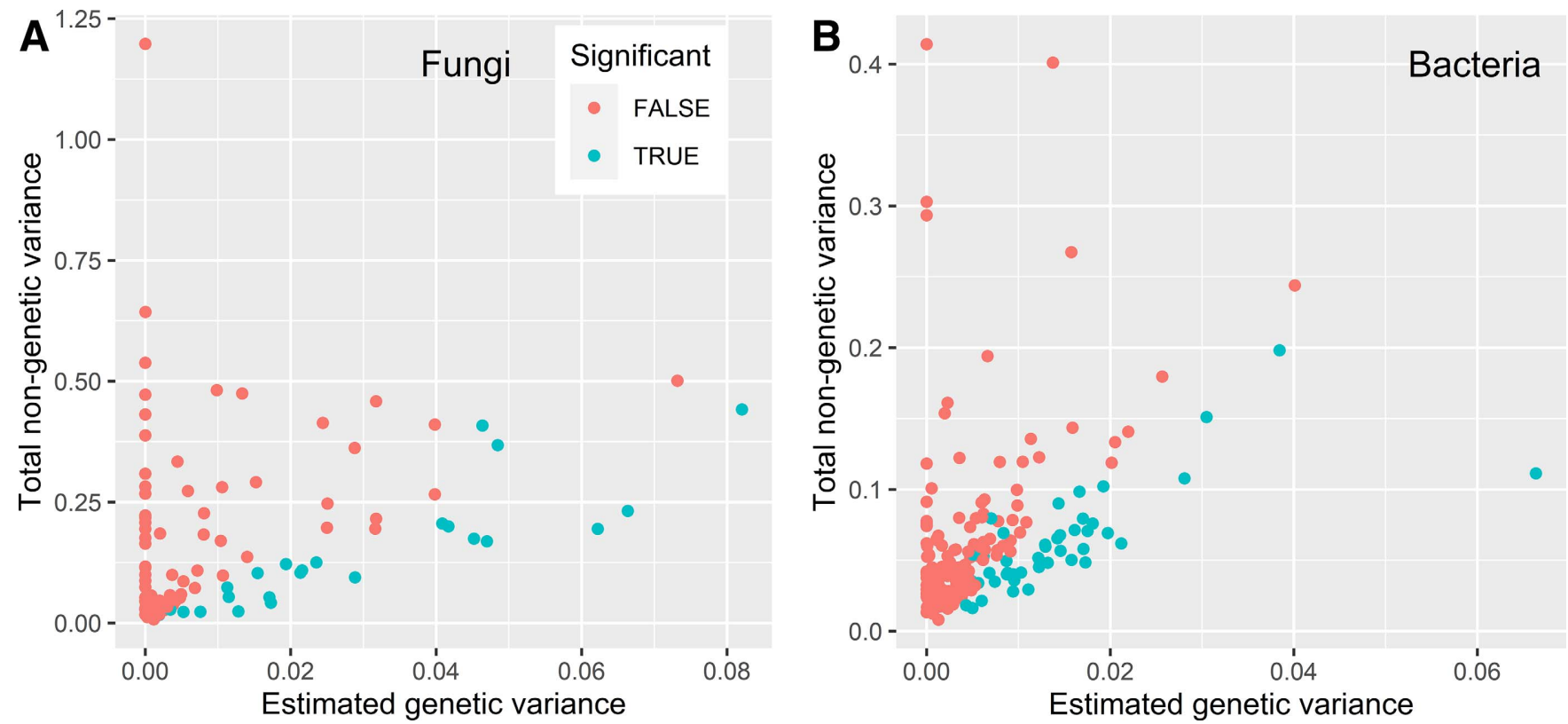

Fig. 4. Estimated genetic variance plotted against the total environmental variance for the top A, 100 fungal and B, 200 bacterial operational taxonomic units based on the median of ratio normalized counts data. The point color indicates whether the genetic variance is statistically significant or not. 
with an average of $11.1 \mathrm{~cm}$ (Fig. 5A). In month 20, 35 trees had cankers in all shoots (Fig. 5C). The two parents did not differ in any of the three canker measurements. In contrast, $\mathrm{F}_{1}$ genotypes differed significantly in average canker size $(P<0.05)$ and canopy coverage (Fig. 5B) and percent shoots with canker in month 20 $(P<0.001)$. The broad-sense heritability was $13.1,23.8$, and $31.9 \%$ for average canker size, percent canopy coverage, and percent shoots with canker at $20 \mathrm{mpi}$, respectively.

Correlation of microbial variables with canker variables. The summary of correlation results is shown in Table 2, with detailed correlation results given in Tables 5 and 6 .

Fungi. Among the top 30 fungal PCs of the qPCR-normalized data, only PC5 scores were correlated with percent canopy coverage in month 20 based on the individual tree data. At the $\mathrm{F}_{1}$ genotypic level, PC5 scores were correlated with percent canopy coverage in month 20, PC7 with canker size, and PC23 with percent shoots cankered. For the MR-normalized data, of the top 80 PCs, only PC4 scores were correlated with percent canopy coverage based on individual trees whereas, based on $F_{1}$ genotypic means, PC3 and P9 were correlated with percent canopy coverage and canker size, respectively.

In total, there were 42 significant correlations of canker variables with 16 fungal OTUs; 33 of these 42 cases were for the MR-normalized data. In 26 of the 42 cases, correlation was at the level of $F_{1}$ genotypic means. In 35, 6, and 1 cases, it involved percent canopy coverage, average canker size, and percent shoots with cankers, respectively. Among these 42 significant correlations,
25 and 17 were of the Pearson and Spearman type, respectively. Correlation coefficient ranged from -0.56 to 0.51 . The magnitude of correlation was greater for genotypic means (from -0.56 to 0.51 ) than for individual trees (from -0.30 to 0.26 ) (Table 5). For several OTUs, the observed correlation was inconsistent; namely, different signs between qPCR- and MR-normalized data (e.g., OTU69) (Table 5), or between individual and genotypic levels (e.g., OTU49) (Table 5), or between Pearson and Spearman correlations (e.g., OTU1896) (Table 5). Nevertheless, several OTUs had consistent correlation patterns, including a group from Entyloma sp. (OTU35) and Pseudoophiobolus rosae (OTU57) (Table 5).

Bacteria. Among the top 30 PCs of the qPCR-normalized data, both Pearson and Spearman correlations of PC11 with percent canopy coverage were significant based on individual trees $(r=-0.24$, -0.32). For the MR-normalized data, PC7 was correlated with percent canopy coverage on individual trees (Pearson: -0.24 , and Spearman: -0.26) (Fig. 6), whereas PC29 was correlated with average canker size (Pearson: 0.27). At the $\mathrm{F}_{1}$ genotypic level, both PC6 and PC10 were correlated with average canker size $(r=-0.50$, 0.47 ) and PC7 with percent canopy coverage (Pearson: -0.44) (Fig. 6)

In total, there were 24 significant correlations of canker variables with 16 bacterial OTUs; 20 of these 24 cases were for the MR-normalized data (Table 6), all with percent canopy cover. All four correlations of qPCR-normalized data were with canker size. In 18 of these 24 cases, correlation was at the $F_{1}$ genotypic level. Half of the correlation was of the Pearson type. Correlation

TABLE 3

Summary of those fungal operational taxonomic units (OTUs) within the top 100 highest counts in the median of ratio normalized counts for which the genetic differences between $F_{1}$ genotypes were statistically significant

\begin{tabular}{|c|c|c|c|}
\hline OTU & Taxonomy (>80\% confidence) & Total reads $(\%)$ & Heritability (\%) \\
\hline OTU29 & Ascomycota & 0.37 & 17.57 \\
\hline OTU3360 & Basidiomycota & 1.30 & 11.00 \\
\hline OTU35 & Entyloma & 0.19 & 17.26 \\
\hline OTU50 & Entyloma calendulae & 0.07 & 24.21 \\
\hline OTU147 & Fungi & 0.07 & 15.67 \\
\hline OTU19 & Fungi & 0.94 & 24.31 \\
\hline OTU40 & Fungi & 0.65 & 10.20 \\
\hline OTU73 & Leptospora & 0.03 & 17.04 \\
\hline OTU76 & Pleosporales & 0.04 & 16.57 \\
\hline OTU57 & Pseudoophiobolus rosae & 0.08 & 22.26 \\
\hline OTU21 & Sporobolomyces roseus & 0.62 & 18.66 \\
\hline OTU26 & Subplenodomus iridicola & 0.49 & 20.57 \\
\hline OTU24 & Symmetrospora & 0.71 & 10.20 \\
\hline OTU310 & Taphrina & 0.04 & 29.12 \\
\hline OTU71 & Taphrina & 0.05 & 13.02 \\
\hline OTU16 & Taphrina carpini & 2.04 & 34.73 \\
\hline OTU15 & Tilletiopsis washingtonensis & 1.40 & 16.54 \\
\hline
\end{tabular}


TABLE 4

Summary of those bacterial operational taxonomic units (OTUs) within the 200 highest counts (median of ratio normalized) for which the genetic differences between $F_{1}$ genotypes were statistically significant

\begin{tabular}{|c|c|c|c|}
\hline OTU & $\begin{array}{c}\text { Taxonomy } \\
(>80 \% \text { confidence })\end{array}$ & $\begin{array}{l}\text { Total reads } \\
(\%)\end{array}$ & $\begin{array}{l}\text { Heritability } \\
\text { (\%) }\end{array}$ \\
\hline OTU68 & Acetobacteraceae & 0.18 & 17.87 \\
\hline OTU16 & Actinobacteria & 1.21 & 8.61 \\
\hline OTU644 & Actinobacteria & 0.19 & 26.27 \\
\hline OTU383 & Alphaproteobacteria & 0.16 & 23.45 \\
\hline OTU105 & Arthrobacter & 0.10 & 21.01 \\
\hline OTU27 & Aurantimonadaceae & 0.67 & 10.49 \\
\hline OTU38 & Aureimonas & 0.48 & 21.04 \\
\hline OTU128 & Bacteria & 0.10 & 14.91 \\
\hline OTU59 & Bacteria & 0.22 & 19.06 \\
\hline OTU813 & Bacteria & 0.11 & 17.52 \\
\hline OTU950 & Bacteria & 0.21 & 18.97 \\
\hline OTU52 & Comamonadaceae & 0.26 & 13.76 \\
\hline OTU135 & Deinococcus & 0.08 & 25.53 \\
\hline OTU18 & Deinococcus & 1.15 & 19.88 \\
\hline OTU124 & Deltaproteobacteria & 0.08 & 16.79 \\
\hline OTU170 & Dyadobacter & 0.10 & 17.86 \\
\hline OTU57 & Enterococcus & 0.20 & 17.64 \\
\hline OTU46 & Erythrobacteraceae & 0.28 & 12.13 \\
\hline OTU2048 & Firmicutes & 0.07 & 19.93 \\
\hline OTU5 & Kineococcus & 2.74 & 14.17 \\
\hline OTU53 & Marmoricola & 0.23 & 14.46 \\
\hline OTU13 & Methylobacteriaceae & 1.28 & 12.11 \\
\hline OTU3 & Methylobacterium & 4.27 & 9.34 \\
\hline OTU462 & Microbacteriaceae & 0.09 & 17.65 \\
\hline OTU504 & Microbacteriaceae & 0.13 & 14.23 \\
\hline OTU37 & Nakamurellales & 0.54 & 17.83 \\
\hline OTU123 & Nocardioides & 0.09 & 18.42 \\
\hline OTU42 & Nocardioides & 0.35 & 10.78 \\
\hline OTU7 & Novosphingobium & 1.86 & 22.73 \\
\hline OTU2625 & Proteobacteria & 0.09 & 21.81 \\
\hline OTU73 & Proteobacteria & 0.19 & 21.27 \\
\hline OTU922 & Proteobacteria & 0.14 & 25.14 \\
\hline OTU85 & Pseudokineococcus & 0.14 & 20.44 \\
\hline OTU100 & Rhodobacteraceae & 0.10 & 17.66 \\
\hline OTU30 & Rhodococcus & 0.70 & 8.12 \\
\hline OTU3169 & Rhodospirillales & 0.08 & 23.89 \\
\hline OTU126 & Roseomonas & 0.08 & 22.19 \\
\hline OTU47 & Roseomonas & 0.32 & 21.44 \\
\hline OTU84 & Roseomonas & 0.14 & 17.45 \\
\hline OTU89 & Roseomonas & 0.13 & 37.34 \\
\hline OTU20 & Sphingomonadaceae & 0.93 & 20.67 \\
\hline OTU114 & Sphingomonas & 0.08 & 15.86 \\
\hline OTU149 & Sphingomonas & 0.08 & 19.25 \\
\hline OTU8 & Sphingomonas & 1.76 & 18.97 \\
\hline OTU49 & Spirosoma & 0.32 & 27.32 \\
\hline OTU99 & Terracoccus & 0.10 & 16.25 \\
\hline OTU60 & Williamsia & 0.24 & 19.93 \\
\hline
\end{tabular}


correlated with the diversity characteristics of the rhizosphere microbiome (Peiffer et al. 2013). The present research is based on an $F_{1}$ population from a specific cross, hence representing genetic variance between the two specific parental genotypes only. The overall low heritability of the phytobiome may be due to strong environmental effects (Clouse and Wagner 2021), including spatial and temporal variation. For instance, we recently demonstrated that, although cultivars differed in their endophytes in leaf scars, orchard locations accounted for much greater variability in endophyte composition (Olivieri et al. 2021a). Plants are only able to recruit those microbial organisms present at a specific site with the recruitment outcome, likely depending on frequencies of available taxa. This
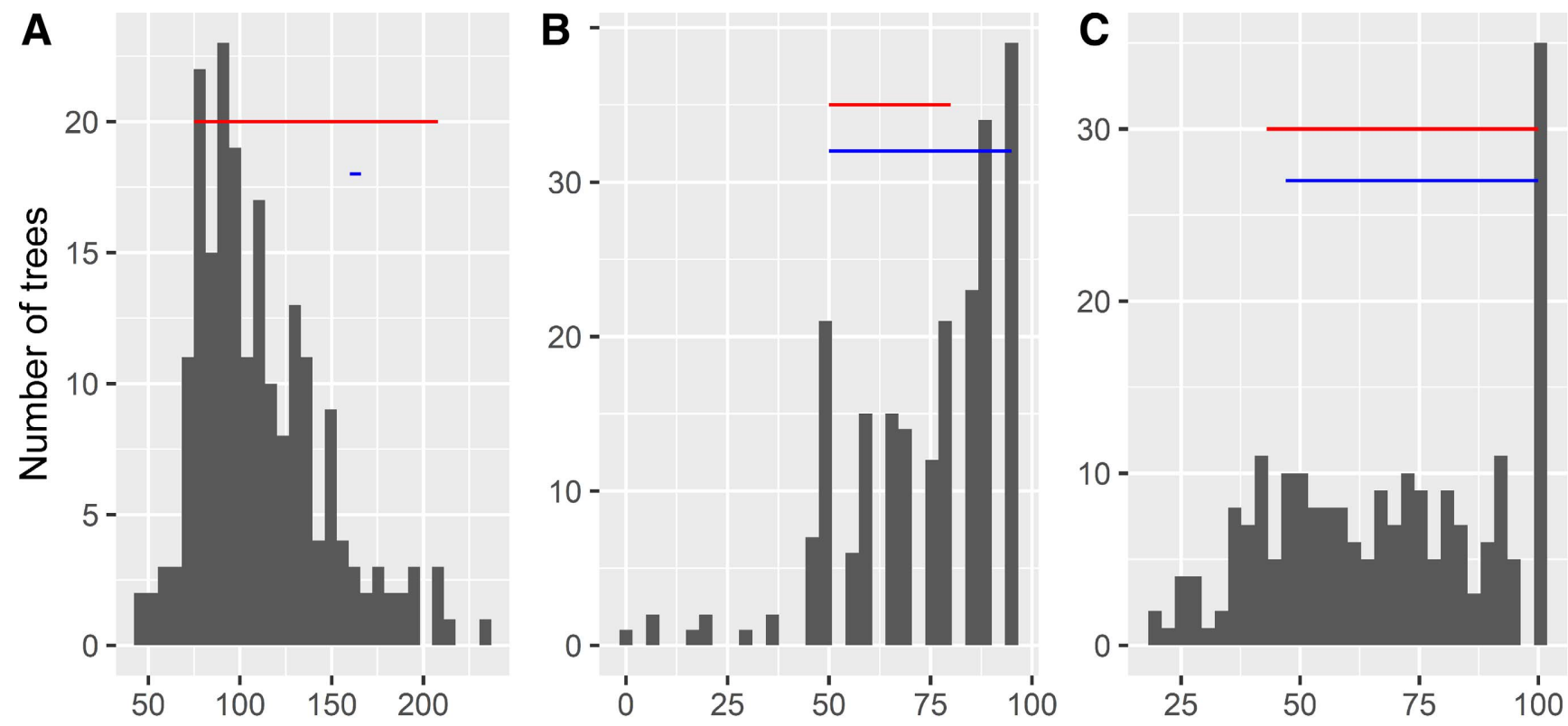

Average canker size $(\mathrm{mm})$ Canopy coverage in month $20 \%$ shoots with canker in month

Fig. 5. Histogram of three canker variables assessed on individual trees. A, Average canker size measured 5, 8, and 11 months postinoculation; B, percent canopy cover; and C, percent shoots with cankers assessed 20 months postinoculation. Blue and red lines indicate the range of values for the female and male parents, respectively.

TABLE 5

Significant Pearson and Spearman (in parentheses) correlation coefficients of canker size, canopy cover and percent shoots with canker 20 months postinoculation with fungal operational taxonomic units (OTUs), with the highest counts based on individual trees or $F_{1}$ genotypic means

\begin{tabular}{|c|c|c|c|c|c|c|c|c|}
\hline \multirow[b]{2}{*}{ OTU ID } & \multirow[b]{2}{*}{ Taxonomy } & \multicolumn{3}{|c|}{ Quantitative PCR normalized data } & \multicolumn{4}{|c|}{ Median of ratio normalized data } \\
\hline & & $\frac{\text { Individual }}{\text { Canopy }}$ & Canker size & Canopy & $\frac{\text { Individual }}{\text { Canopy }}$ & Canker size & Canopy & Shoots (\%) \\
\hline OTU143 & Dothideomycetes & - & - & -0.43 & - & - & - & - \\
\hline OTU35 & Entyloma & - & $(-0.47)$ & - & - & $-0.50(-0.55)$ & - & - \\
\hline OTU59 & Exobasidiomycetes & - & - & - & - & - & $-0.45(-0.42)$ & - \\
\hline OTU43 & Fungi & - & $(-0.52)$ & - & - & $-0.53(-0.56)$ & - & - \\
\hline OTU6 & Fungi & -0.28 & - & - & $-0.24(-0.22)$ & - & - & - \\
\hline OTU69 & Fungi & - & - & -0.50 & - & - & 0.47 & - \\
\hline OTU17 & Neosetophoma & - & - & - & $0.24(0.23)$ & - & - & - \\
\hline OTU20 & Phaeosphaeriaceae & - & - & -0.41 & - & - & -0.40 & - \\
\hline OTU21 & Sporobolomyces roseus & - & - & - & $-0.21(0.26)$ & - & $0.51(0.50)$ & - \\
\hline OTU1896 & Vishniacozyma & - & - & - & $(-0.28)$ & - & $-0.41(0.44)$ & -0.48 \\
\hline OTU3 & Vishniacozyma & - & - & - & - & - & $0.40(0.42)$ & - \\
\hline OTU18 & Vishniacozyma carnescens & - & - & - & $0.26(0.21)$ & - & - & - \\
\hline
\end{tabular}


potential large difference in aerosol microbiome over distance may also partially explain the block effects on endophytes observed in the present study.

Only a few specific microbial groups were significantly correlated with canker size or percent canopy cover. Interestingly, all significant correlations with bacterial groups indicated that higher relative abundance of these groups is associated with less canker development, mostly with better canopy coverage and, in a few instances, negatively with canker size. In contrast, there is no such consistency in the correlation of fungal groups with canker development: both positive and negative correlation with canker development were observed. Moreover, the inconsistency exists even for the same fungal group across different canker variables or parametric or rank correlations, or at the tree or genotypic levels. Reasons

TABLE 6

Significant Pearson and Spearman (in parentheses) correlation coefficients of canker size, canopy cover and percent shoots with canker 20 months postinoculation with most abundant bacterial operational taxonomic units (OTUs) based on individual trees or $F_{1}$ genotypic means

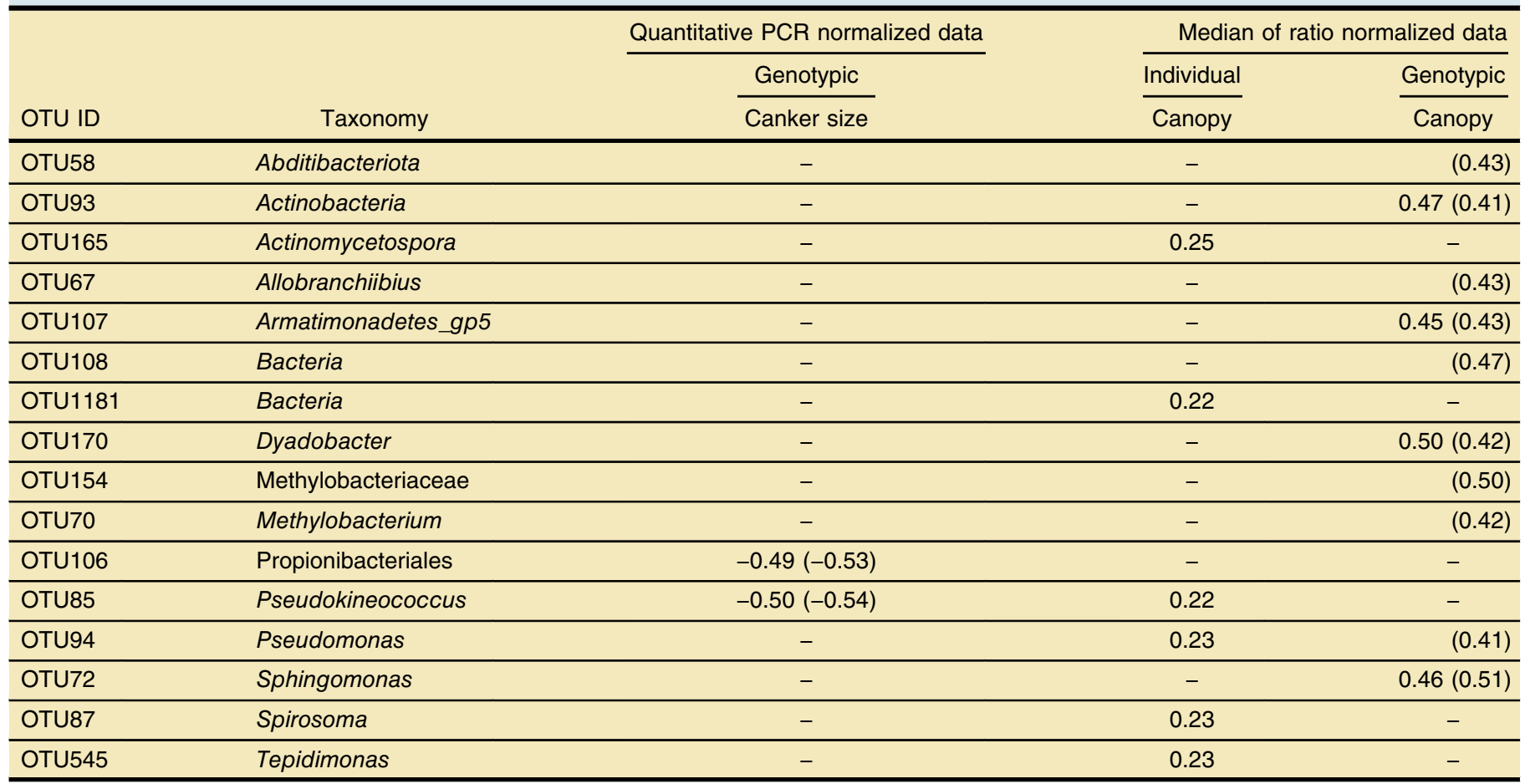

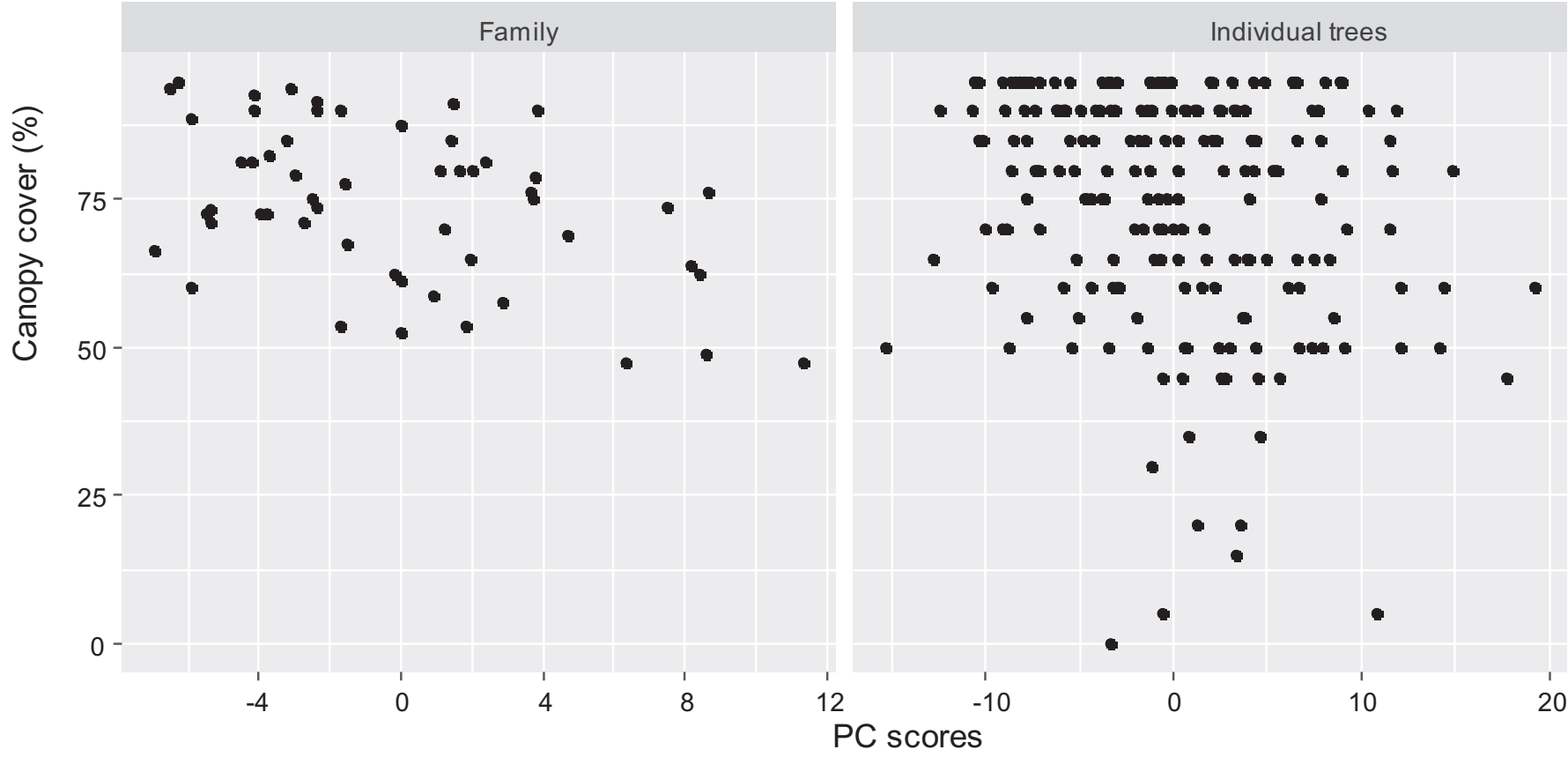

Fig. 6. Principal component 7 (PC7) scores of the median of ratio normalized bacterial operational taxonomic unit data plotted against canopy coverage 20 months postinoculation. 
for these observed differences are not obvious. As discussed above, recruitment of endophytes may be considerably influenced by the local aerosol microbiome, not necessarily affected by tree genotypes. Thus, individual trees may differ greatly in relative abundance for some endophytes not because of genetics but because of variabilities in the aerosol microbiome. If these microbes have direct or indirect effects on $N$. ditissima, we may expect inconsistent correlations at the individual and genotypic levels. This may be further complicated by possible differences in the stability or persistence of individual endophytes in the leaf scar tissues at a given endophyte background. For example, fungal endophyte composition might be more easily influenced by differences in aerosol microbiome or external conditions than bacteria. Communities of endophytic fungi assembling in several plant species depend significantly on proximity to the inoculum source as well as the identity of the plant species (Ricks and Koide 2019). It is not known what fraction of the endophytic bacterial microbiome is dispersed via the atmosphere or originated from the atmosphere (Frank et al. 2017). Further research is needed to understand the relative importance of sources for plant endophytes and the effects of biotic and abiotic factors on the stability of the endophyte microbiome over time before we can amend the phytobiome with a reasonable level of predictability to improve crop performance.

Most of those bacterial groups with significant correlations with canker development cannot be classified into genus. Dyadobacter isolates are one of the major cohorts of bacteria in the plant phyllosphere (Delmotte et al. 2009; Reisberg et al. 2012) but what role Dyadobacter spp. play in these communities is currently unknown. The relative abundance of a Pseudomonas OTU (OTU94) and Sphingomonas OTU (OTU72) is positively correlated with canopy coverage in the present study. A Pseudomonas strain isolated form apple endophytes was shown to be antagonistic toward $N$. ditissima in in vitro tests (Liu et al. 2020). A seedendophytic strain (Sphingomona melonis ZJ26) confers rice with disease resistance against a bacterial pathogen and is vertically transmitted among plant generations via their seeds (Matsumoto et al. 2021). A couple of Sphingomonas groups were more abundant in canker-susceptible cultivars than in resistant cultivars (Olivieri et al. 2021a); however, that particular study did not consider the actual canker severity. Methylobacterium normally resides in soil and water but has also been identified as a contaminant of DNA extraction kit reagents, which may lead to its erroneous appearance in microbiota or metagenomic datasets (Salter et al. 2014). Given that these bacterial groups are mostly correlated with canopy cover but not with canker size, we may speculate that they may not be effective as direct competitors of $N$. ditissima but, rather, improve plant tolerance against consequences on plant development due to canker.

Only 3 of the 16 fungal groups with significant correlations with canker development can be classified into species: Vishniacozyma carnescens $(\mathrm{syn}=$ Cryptococcus carnescens), Pseudoophiobolus rosae, and Sporobolomyces roseus. Of all 16 groups, only $V$. carnescens is associated with reduced canker damage (but only at the individual tree level); unfortunately, there is no published information on its ecology in relation to plants. An Entyloma sp. (OTU35) is positively correlated with canker tolerance but Entyloma is a genus of plant-pathogenic smut fungi. Thus far, these fungal groups have not been associated with a direct antagonistic effect against $N$. ditissima (Liu et al. 2020) or associated with cultivars with differential susceptibility to $N$. ditissima (Olivieri et al. 2021a). A couple of groups appear to facilitate canker development, including P. rosae and Exobasidiomycetes (OTU59). Exobasidiomycetes are a class of fungi sometimes associated with galls of plant tissues. Further metagenome sequencing and isolation combined with challenging assays against $N$. ditissima are needed to make further progress in this area.

In summary, some components of the apple endophyte microbiome as well as individual microbial groups around leaf scar tissues are partially controlled genetically by apple genotypes. Several microbial groups had significant correlation with canker development. Bacterial groups appear to be positively associated with canker tolerance. On the other hand, a few fungal groups may facilitate canker development whereas a few others may compete with the canker pathogen. The present results may be used to inform targeted approaches to further research in biocontrol of $N$. ditissima with specific microbes and breeding for resistance.

\section{LITERATURE CITED}

Bates, D., Mächler, M., Bolker, B. M., and Walker, S. C. 2015. Fitting linear mixed-effects models using lme4. J. Stat. Softw. 67:1-48.

Benjamini, Y., and Hochberg, Y. 1995. Controlling the false discovery rate: A practical and powerful approach to multiple testing. J. Roy. Stat. Soc. B 57:289-300.

Bus, V. G. M., Scheper, R. W. A., Walter, M., Campbell, R. E., Kitson, B., Turner, L., Fisher, B. M., Johnston, S. L., Wu, C., Deng, C. H., Singla, G., Bowatte, D., Jesson, L. K., Hedderley, D. I., Volz, R. K., Chagné, D., and Gardiner, S. E. 2019. Genetic mapping of the European canker (Neonectria ditissima) resistance locus Rnd1 from Malus 'Robusta 5'. Tree Genet. Genomes 15:25.

Clouse, K. M., and Wagner, M. R. 2021. Plant genetics as a tool for manipulating crop microbiomes: Opportunities and challenges. Front. Bioeng. Biotechnol. 9.

Cole, J. R., Wang, Q., Fish, J. A., Chai, B., McGarrell, D. M., Sun, Y., Brown, C. T., Porras-Alfaro, A., Kuske, C. R., and Tiedje, J. M. 2014. Ribosomal Database Project: Data and tools for high throughput rRNA analysis. Nucleic Acids Res. 42:D633-D642.

Delmotte, N., Knief, C., Chaffron, S., Innerebner, G., Roschitzki, B., Schlapbach, R., von Mering, C., and Vorholt, J. A. 2009. Community proteogenomics reveals insights into the physiology of phyllosphere bacteria. Proc. Natl. Acad. Sci. U.S.A. 106:16428-16433.

Dini-Andreote, F. 2020. Endophytes: The second layer of plant defense. Trends Plant Sci. 25:319-322.

Dixon, P. 2003. VEGAN, a package of R functions for community ecology. J. Veg. Sci. 14:927-930.

Edgar, R. C. 2013. UPARSE: Highly accurate OTU sequences from microbial amplicon reads. Nat. Methods 10:996-998.

Edgar, R. C., and Flyvbjerg, H. 2015. Error filtering, pair assembly and error correction for next-generation sequencing reads. Bioinformatics 31:34763482.

Frank, A. C., Guzmán, J. P. S., and Shay, J. E. 2017. Transmission of bacterial endophytes. Microorganisms 5:70.

Gómez-Cortecero, A., Saville, R. J., Scheper, R. W. A., Bowen, J. K., de Medeiros, H. A., Kingsnorth, J., Xu, X., and Harrison, R. J. 2016. Variation in host and pathogen in the Neonectria/Malus interactions; towards an understanding of the genetic basis of resistance to European canker. Front. Plant Sci. 7.

Hirakue, A., and Sugiyama, S. 2018. Relationship between foliar endophytes and apple cultivar disease resistance in an organic orchard. Biol. Control 127:139-144.

Khare, E., Mishra, J., and Arora, N. K. 2018. Multifaceted interactions between endophytes and plant: Developments and prospects. Front. Microbiol. 9.

Kõljalg, U., Nilsson, R. H., Abarenkov, K., Tedersoo, L., Taylor, A. F. S., Bahram, M., Bates, S. T., Bruns, T. D., Bengtsson-Palme, J., Callaghan, T. M., Douglas, B., Drenkhan, T., Eberhardt, U., Dueñas, M., Grebenc, T., Griffith, G. W., Hartmann, M., Kirk, P. M., Kohout, P., Larsson, E., Lindahl, B. D., Lücking, R., Martín, M. P., Matheny, P. B., Nguyen, N. H., Niskanen, T., Oja, J., Peay, K. G., Peintner, U., Peterson, M., Põldmaa, K., Saag, L., Saar, I., Schüßler, A., Scott, J. A., Senés, C., Smith, M. E., Suija, A., Taylor, D. L., Telleria, M. T., Weiss, M., and Larsson, K.-H. 2013. Towards a unified paradigm for sequence-based identification of fungi. Mol. Ecol. 22:5271-5277. 
Liu, J., Abdelfattah, A., Norelli, J., Burchard, E., Schena, L., Droby, S., and Wisniewski, M. 2018. Apple endophytic microbiota of different rootstock/scion combinations suggests a genotype-specific influence. Microbiome 6:18

Liu, J., Ridgway, H. J., and Jones, E. E. 2020. Apple endophyte community is shaped by tissue type, cultivar and site and has members with biocontrol potential against Neonectria ditissima. J. Appl. Microbiol. 128:1735-1753.

Love, M. I., Huber, W., and Anders, S. 2014. Moderated estimation of fold change and dispersion for RNA-seq data with DESeq2. Genome Biol. 15:550.

Matsumoto, H., Fan, X., Wang, Y., Kusstatscher, P., Duan, J., Wu, S., Chen, S., Qiao, K., Wang, Y., Ma, B., Zhu, G., Hashidoko, Y., Berg, G., Cernava, T., and Wang, M. 2021. Bacterial seed endophyte shapes disease resistance in rice. Nat. Plants 7:60-72.

Olivieri, L., Saville, R. J., Gange, A. C., and Xu, X. 2021a. Apple endophyte community in relation to location, scion and rootstock genotypes and susceptibility to European canker. FEMS Microbiol. Ecol. 97:fiab131.

Olivieri, L., Saville, R. J., Gange, A. C., and Xu, X. 2021b. Limited asymptomatic colonisation of apple tree shoots by Neonectria ditissima following infection of leaf scars and pruning wounds. Plant Pathol. 70: 1838-1849.

Omomowo, O. I., and Babalola, O. O. 2019. Bacterial and fungal endophytes: Tiny giants with immense beneficial potential for plant growth and sustainable agricultural productivity. Microorganisms 7:481.

Peiffer, J. A., Spor, A., Koren, O., Jin, Z., Tringe, S. G., Dangl, J. L., Bicl;er, E. S., and Ley, R. E. 2013. Diversity and heritability of the maize rhizosphere microbiome under field conditions. Proc. Natl. Acad. Sci. U.S.A. $110: 6548-6553$.

R Core Development Team. 2019. R: A Language and Environment for Statistical Computing. R Foundation for Statistical Computing, Vienna, Austria. https://www.R-project.org/
Reisberg, E. E., Hildebrandt, U., Riederer, M., and Hentschel, U. 2012. Phyllosphere bacterial communities of trichome-bearing and trichomeless Arabidopsis thaliana leaves. Antonie Leeuwenhoek 101:551-560.

Ricks, K. D., and Koide, R. T. 2019. The role of inoculum dispersal and plant species identity in the assembly of leaf endophytic fungal communities. PLoS One 14:e0219832.

Salter, S. J., Cox, M. J., Turek, E. M., Calus, S. T., Cookson, W. O., Moffatt, M. F., Turner, P., Parkhill, J., Loman, N. J., and Walker, A. W. 2014. Reagent contamination can critically impact sequence-based microbiome analyses. BMC Biol. 12:87.

Saville, R. J., and Olivieri, L. 2019. Fungal diseases of fruit: Apple cankers in Europe. Pages 59-84 in: Integrated Management of Diseases and Insect Pests of Tree Fruit. X. Xu and M., Fountain, eds. Burleigh Dodds Science Publishing, Cambridge, U.K.

Tkacz, A., Hortala, M., and Poole, P. S. 2018. Absolute quantitation of microbiota abundance in environmental samples. Microbiome 6:110.

Wagner, M. R., Lundberg, D. S., del Rio, T. G., Tringe, S. G., Dangl, J. L., and Mitchell-Olds, T. 2016. Host genotype and age shape the leaf and root microbiomes of a wild perennial plant. Nat. Commun. 71:12151.

Wagner, M. R., Roberts, J. H., Balint-Kurti, P., and Holland, J. B. 2020. Heterosis of leaf and rhizosphere microbiomes in field-grown maize. New Phytol. 228:1055-1069.

Weber, R. W. S. 2014. Biology and control of the apple canker fungus Neonectria ditissima (syn. N. galligena) from a Northwestern European perspective (Biologie und Kontrolle des Obstbaumkrebs-Erregers Neonectria ditissima (Syn. N. galligena) aus der Perspektive Nordwesteuropas). Erwerbs-Obstbau 56:95-107.

Wei, F., Zhao, L., Xu, X., Feng, H., Shi, Y., Deakin, G., Feng, Z., and Zhu, H. 2019. Cultivar-dependent variation of the cotton rhizosphere and endosphere microbiome under field conditions. Front. Plant Sci. 10. 